\title{
THE SOLUTION OF DUAL COSINE SERIES BY THE USE OF ORTHOGONALITY RELATIONS
}

\author{
by B. NOBLE and J. R. WHITEMAN $\dagger$ \\ (Received 31st December 1968)
}

\section{Introduction}

In this paper expressions are derived for the unknown coefficients in the dual cosine series:

$$
\begin{gathered}
\frac{1}{2} a_{0} \cos \frac{x}{2}+\sum_{n=1}^{\infty} a_{n} \cos \left(n+\frac{1}{2}\right) x=f_{1}(x), \quad 0<x<c, \\
\frac{1}{2} \alpha a_{0} \cos \frac{x}{2}+\sum_{n=1}^{\infty} a_{n} \frac{n(n+1)}{2 n+1} \cos \left(n+\frac{1}{2}\right) x=f_{2}(x), \quad c<x<\pi,
\end{gathered}
$$

where $\alpha$ is a given constant, and $f_{1}(x)$ and $f_{2}(x)$ are prescribed functions.

The method is an extension of that of a previous paper, see Noble and Whiteman (5), and is a generalization of the method of multiplying-factors described by Noble (4), Section 3, or the equivalent fractional integration technique of Erdélyi and Sneddon (2). As far as we know, the solution of the series (1) and (2) is completely new. In the analysis that follows, which is purely formal, two orthogonality relations for Legendre polynomials are needed. These and other required results are stated in Section 3.

\section{Technique for solution}

The method outlined here will be used directly in Section 3 to find the coefficients $a_{n}$ in (1) and (2). As it can also be used with slight modification to solve some well-known dual series, the details are given in general terms. The dual series (1) and (2) have the form:

$$
\begin{aligned}
& \sum_{n=0}^{\infty} a_{n} \alpha(n) \phi_{n}(x)=f_{1}(x), \quad 0<x<c, \\
& \sum_{n=0}^{\infty} a_{n} \beta(n) \phi_{n}(x)=f_{2}(x), \quad c<x<\pi .
\end{aligned}
$$

In order to find the $a_{n}$ we multiply (3) by $p(\theta, x)$ and (4) by $q(\theta, x)$, where the forms of $p$ and $q$ will be specified later, and integrate the respective results from 0 to $\theta(0 \leqq \theta \leqq c)$ and $\theta$ to $\pi(c \leqq \theta \leqq \pi)$ with respect to $x$. After change in the order of summation and integration, the resulting expressions can be

$\dagger$ Sponsored by the Mathematics Research Center, United States Army, Madison, Wisconsin, under contract No. DA-31-124-ARO-D-462. 
written as

where

$$
\begin{aligned}
& \sum_{n=0}^{\infty} a_{n} \alpha(n) \xi_{n}(\theta)=\int_{0}^{\theta} p(\theta, x) f_{1}(x) d x, \\
& \sum_{n=0}^{\infty} a_{n} \beta(n) \eta_{n}(\theta)=\int_{\theta}^{\pi} q(\theta, x) f_{2}(x) d x,
\end{aligned}
$$

and

$$
\xi_{n}(\theta)=\int_{0}^{\theta} p(\theta, x) \phi_{n}(x) d x
$$

$$
\eta_{n}(\theta)=\int_{\theta}^{\pi} q(\theta, x) \phi_{n}(x) d x
$$

and the functions $\xi_{n}(\theta)$ and $\eta_{n}(\theta)$ are connected by the relations

$$
\frac{d \xi_{n}(\theta)}{d \theta}=\frac{n(n+1)}{2 n+1} \frac{\eta_{n}(\theta)}{\sin \theta}
$$

and

$$
\frac{d \eta_{n}(\theta)}{d \theta}=-(2 n+1) \sin \theta \xi_{n}(\theta)
$$

Equations (3) and (4) contain $a_{0}$ terms which do not have the form of the remainder of the series. These are assimilated in the right-hand sides of the equations, and we write

and

$$
\tilde{f}_{1}(x)=f_{1}(x)-\frac{1}{2} a_{0} \cos \frac{1}{2} x \text {. }
$$

$$
\tilde{f}_{2}(x)=f_{2}(x)-\frac{1}{2} \alpha a_{0} \cos \frac{1}{2} x \text {. }
$$

Equation (5) is now multiplied by $\xi_{m}(\theta) \sin \theta$ and the result is integrated from 0 to $c$ with respect to $\theta$. Assuming that $\xi_{n}(0)=0$, so that the end contributions vanish, and that (5) holds for $\theta=c$, we have

$$
\sum_{n=1}^{\infty} a_{n} \beta(n) \int_{0}^{c} \eta_{n}(\theta) \eta_{m}(\theta) \frac{d \theta}{\sin \theta}=\int_{0}^{c} \eta_{m}(\theta) d \theta \frac{d}{d \theta} \int_{0}^{\theta} p(\theta, x) f_{1}(x) d x .
$$

In the right-hand side of (9) the inner integral involving the $a_{0}$ term is a constant, so that the $f_{1}(x)$ here may be written as $f_{1}(x)$. It follows immediately from (6) that

$$
\sum_{n=1}^{\infty} a_{n} \beta(n) \int_{c}^{\pi} \eta_{n}(\theta) \eta_{m}(\theta) \frac{d \theta}{\sin \theta}=\int_{c}^{\pi} \eta_{m}(\theta) \frac{d \theta}{\sin \theta} \int_{\theta}^{n} q(\theta, x) f_{2}(x) d x
$$

Equations (9) and (10) are combined, and the orthogonality relation

$$
\int_{0}^{\pi} \eta_{n}(\theta) \eta_{m}(\theta) \frac{d \theta}{\sin \theta}=\frac{2 \delta_{m n}}{\beta(n)}
$$

where $\delta_{m n}$ is the Kronecker delta, is used so that

$a_{n}=\frac{1}{2} \int_{0}^{c} \eta_{n}(\theta) d \theta \frac{d}{d \theta} \int_{0}^{\theta} p(\theta, x) f_{1}(x) d x+\frac{1}{2} \int_{c}^{\pi} \eta_{n}(\theta) \frac{d \theta}{\sin \theta} \int_{\theta}^{\pi} q(\theta, x) \tilde{f}_{2}(x) d x$. 


\section{Mathematical formulae}

Two well-known relations for Legendre polynomials and their derivatives are

$$
P_{n}(\cos \theta)=-\frac{1}{(2 n+1) \sin \theta} \frac{d}{d \theta}\left[P_{n+1}(\cos \theta)-P_{n-1}(\cos \theta)\right]
$$

and

$$
P_{n+1}(\cos \theta)-P_{n-1}(\cos \theta)=\frac{2 n+1}{n(n+1)} \sin \theta \frac{d}{d \theta} P_{n}(\cos \theta),
$$

(see Magnus and Oberhettinger (3)). When the orthogonality relation

$$
\int_{0}^{\pi} P_{n}(\cos \theta) P_{m}(\cos \theta) \sin \theta d \theta=\frac{2 \delta_{m n}}{2 n+1}
$$

is integrated by parts, the use of (13) and (14) gives

$$
\begin{aligned}
\int_{0}^{n}\left[P_{n+1}(\cos \theta)-P_{n-1}(\cos \theta)\right]\left[P_{m+1}(\cos \theta)-P_{m-1}(\cos \theta)\right] & \frac{d \theta}{\sin \theta} \\
& =\frac{2(2 n+1)}{n(n+1)} \delta_{m n} .
\end{aligned}
$$

Mehler's integrals for Legendre polynomials are

$$
P_{n}(\cos \theta)=\frac{\sqrt{2}}{\pi} \int_{0}^{\theta} \frac{\cos \left(n+\frac{1}{2}\right) x d x}{(\cos x-\cos \theta)^{\frac{1}{2}}}=\frac{\sqrt{2}}{\pi} \int_{\theta}^{\pi} \frac{\sin \left(n+\frac{1}{2}\right) x d x}{(\cos \theta-\cos x)^{\frac{1}{2}}}
$$

(Magnus and Oberhettinger (3)), and from the second of these we obtain

$$
P_{n+1}(\cos \theta)-P_{n-1}(\cos \theta)=\frac{2 \sqrt{2}}{\pi} \int_{\theta}^{\pi} \frac{\cos \left(n+\frac{1}{2}\right) x \sin x d x}{(\cos \theta-\cos x)^{\frac{1}{2}}} .
$$

\section{Solution of the series}

The coefficients $a_{n}$ in the dual series

$$
\begin{gathered}
\frac{1}{2} a_{0} \cos \frac{x}{2}+\sum_{n=1}^{\infty} a_{n} \cos \left(n+\frac{1}{2}\right) x=f_{1}(x), \quad 0<x<c, \\
\frac{1}{2} \alpha a_{0} \cos \frac{x}{2}+\sum_{n=1}^{\infty} a_{n} \frac{n(n+1)}{2 n+1} \cos \left(n+\frac{1}{2}\right) x=f_{2}(x), \quad c<x<\pi,
\end{gathered}
$$

are found using the technique outlined in Section 2. Referring back to that method, we choose

and

$$
p(\theta, x)=\frac{\sqrt{2}}{\pi(\cos x-\cos \theta)^{\frac{1}{2}}}
$$

$$
q(\theta, x)=\frac{2 \sqrt{2} \sin x}{\pi(\cos \theta-\cos x)^{\frac{1}{2}}}
$$

E.M.S.-D 
so that from (17) and (18)

and

$$
\xi_{n}(\theta)=P_{n}(\cos \theta)
$$

$$
\eta_{n}(\theta)=\left[P_{n+1}(\cos \theta)-P_{n-1}(\cos \theta)\right] .
$$

The orthogonality relation (11) is thus identical with (16), so that from (12)

$a_{n}=\frac{1}{\sqrt{2} \pi} \int_{0}^{c}\left[P_{n+1}(\cos \theta)-P_{n-1}(\cos \theta)\right] d \theta \cdot \frac{d}{d \theta} \int_{0}^{\theta} \frac{f_{1}(x) d x}{(\cos x-\cos \theta)^{\frac{1}{2}}}$

$+\frac{\sqrt{2}}{\pi} \int_{c}^{\pi}\left[P_{n+1}(\cos \theta)-P_{n-1}(\cos \theta)\right] \frac{d \theta}{\sin \theta} \int_{\theta}^{\pi} \frac{\left(f_{2}(x)-\frac{1}{2} x a_{0} \cos \frac{1}{2} x\right) \sin x d x}{(\cos \theta-\cos x)^{\frac{1}{2}}}$

for $n=1,2, \ldots$.

The remaining coefficient $a_{0}$ is found by substituting (25) for $a_{n}$ in (19), and then using the relation

$$
\begin{aligned}
\sum_{n=1}^{\infty}\left[P_{n+1}(\cos \theta)-P_{n-1}(\cos \theta)\right] \cos (n & \left.+\frac{1}{2}\right) x \\
& =\frac{\sqrt{2} H(x-\theta) \sin x}{(\cos \theta-\cos x)^{\frac{1}{2}}}-(1+\cos \theta) \cos \frac{1}{2} x,
\end{aligned}
$$

$H(x-\theta)$ being the Heaviside unit function (see Sneddon (6)). After some simplification we obtain

$$
\begin{aligned}
& a_{0}=\frac{1}{K}\left\{\frac{1}{\sqrt{2} \pi} \int_{0}^{c}(1+\cos \theta) d \theta \cdot \frac{d}{d \theta} \int_{0}^{\theta} \frac{f_{1}(x) d x}{(\cos x-\cos \theta)^{\frac{1}{2}}}\right. \\
& \left.+\frac{\sqrt{2}}{\pi} \int_{c}^{\pi} \cot \frac{1}{2} \theta d \theta \int_{\theta}^{\pi} \frac{f_{2}(x) d x}{(\cos \theta-\cos x)^{\frac{1}{2}}}\right\}
\end{aligned}
$$

where $K=\frac{1}{2}\left(1-\alpha \cos ^{2} \frac{1}{2} c-2 \alpha \log \sin \frac{1}{2} c\right)$.

The method of Section 2 can be used to solve the much-studied series

$$
\begin{gathered}
\sum_{n=0}^{\infty} a_{n}(2 n+1)^{p} \cos \left(n+\frac{1}{2}\right) x=f_{1}(x), \quad 0<x<c, \\
\sum_{n=0}^{\infty} a_{n} \cos \left(n+\frac{1}{2}\right) x=f_{2}(x), \quad c<x<\pi,
\end{gathered}
$$

where $p= \pm 1$. These series are those of equations (3) and (4) when

and

$$
\alpha(n)=(2 n+1)^{p}
$$

$$
\beta(n)=1 \text {. }
$$

For the case $p=1$ our method gives the result of Srivastav (7). When in addition $f_{2}(x)=0$, it gives that of Tranter (8). For the case $p=-1$ we obtain an expression for $a_{n}$, which for $f_{2}(x)=0$ is that of Tranter (8). However, for general $f_{1}(x)$ and $f_{2}(x)$ we have been unable to transform this into that given by Srivastav (7), which was later corrected by Cooke (1). 


\section{REFERENCES}

(1) J. C. CookE, Note on a pair of dual trigonometrical series, Glasgow Math. J. 9 (1968), 30-35.

(2) A. ERdÉlyi and I. N. SNedDon, Fractional integration and dual integral equations, Canad. J. Math. 14 (1962), 685-693.

(3) W. Magnus and F. Oberhettinger, Special Functions of Mathematical Physics (Chelsea, New York, 1949).

(4) B. Noble, Some dual series equations involving Jacobi polynomials, Proc. Cambridge Philos. Soc. 59 (1963), 363-371.

(5) B. Noble and J. R. Whrteman, Solution of dual trigonometrical series using orthogonality relations (Mathematics Research Center, Technical Summary Report \# 890, Madison, 1968).

(6) I. N. Sneddon, Mixed Boundary Value Problems in Potential Theory (North Holland, Amsterdam, 1966).

(7) R. P. SRtvastav, Dual series relations-III. Dual relations involving trigonometrical series, Proc. Roy. Soc. Edinburgh, Sect. A 66 (1964), 173-184.

(8) C. J. Tranter, An improved method for dual trigonometrical series, Proc. Glasgow Math. Assoc. 6 (1964), 136-140.

Mathematics Research Center

UNIVERSITY OF WISCONSIN 